International Journal of Medical Anesthesiology 2020; 3(4): 78-82

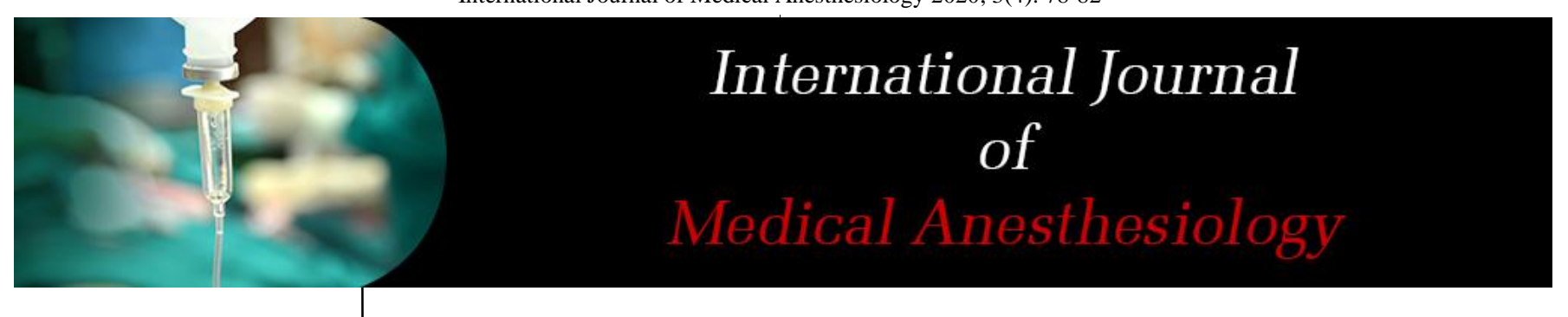

E-ISSN: 2664-3774

P-ISSN: 2664-3766

Www.anesthesiologypaper.com

IJMA 2020; 3(4): 78-82

Received: 26-08-2020

Accepted: 05-10-2020

Dr. CR Murali Govardhan

Associate Professor,

Department of

Anaesthesiology, MNR

Medical College\& Hospital,

Sangareddy, Telangana, India
Corresponding Author: Dr. CR Murali Govardhan Associate Professor, Department of

Anaesthesiology, MNR

Medical College\& Hospital,

Sangareddy, Telangana, India

\section{Efficacy of dexmedetomidine on postoperative analgesia \& sedation in children undergoing adenotonsillectomy}

\author{
Dr. CR Murali Govardhan
}

DOI: $\underline{\text { https://doi.org/10.33545/26643766.2020.v3.i4b.168 }}$

\begin{abstract}
Background: Adenotonsillectomy is a common surgical procedure in pediatric population, but postoperative pain management is a challenging concern for anaesthetist. The present study was designed to assess the efficacy of dexmedetomidine on post-operative sedation and analgesia in paediatric cases undergoing adenotonsillectomy.

Materials and methods: A total 80 pediatric cases undergoing adenotonsillectomy between age group 4-9 years belong to ASA grade I and II were recruited. Study consists of two groups i.e. group 1 with $1 \mathrm{mcg} / \mathrm{kg}$ Dexmeditomidine and group 2 with $2 \mathrm{mcg} / \mathrm{kg}$ fentanyl. Parameters like heart rate, systolic blood pressure (SBP), diastolic blood pressure (DBP), requirement of post-operative analgesia and sedation assessed by Ramsay sedation score \& CHEOPS score and emergence agitation were assessed. Results: The mean heart rate was significant between two study groups at the beginning, $5 \mathrm{~min}, 15$ min, 20 min and at $25 \mathrm{~min}(p<0.005)$. The mean SBP in group 1 was high at the beginning, later the values were decreased when compared group 2. However, the mean DBP in group 1 was low when compared to the group 2 at all-time intervals. The mean Ramsay sedation score difference was statistically significant among two study groups at the beginning, $5 \mathrm{~min}, 10 \mathrm{~min}, 15 \mathrm{~min}$ and $45 \mathrm{~min}$ $(p<0.005)$. The requirement of additional rescue doses were noticed more in group 2.

Conclusion: Dexmedetomidine was effective in maintaining intraoperative heart rate, systolic blood pressure and diastolic blood pressure. Dexmedetomidine is an effective and safe analgesic substitute to fentanyl intra-operatively and reduces the requirement of postoperative rescue opioid. Dexmedetomidine was more effective in preventing emergence agitation, avoiding severe pain and reducing incidence of postoperative nausea and vomiting.
\end{abstract}

Keywords: Adenotonsillectomy, dexmedetomidine, ramsay sedation score, emergence agitation, heart rate

\section{Introduction}

Adenotonsillectomy is a hasty, but agonizing surgical procedure carried out in children ${ }^{[1]}$. Posttonsillectomy pain management is a major challenging concern for anaesthetist because of post-operative pain affects analgesic consumption, duration of hospital stay and often associated with high incidences of anxiety, obstructive symptoms and hypoxemia ${ }^{[2]}$.

Dexmedetomidine is a highly potent $\alpha 2$ adrenoceptoragonist, has sedative, analgesic, anxiolytic and opioid sparing properties with or without respiratory depression ${ }^{[3,4]}$. Studies have shown that dexmedetomidine is an effective drug in the post-operative pain management in a pediatric population undergoing adenotonsillectomy ${ }^{[5]}$. Dexmedetomidine is an effective adjunct to reduce the incidence and severity post-operative emergence agitation/delirium and analgesic requirement ${ }^{[6,7]}$. Studies have shown that dexmedetomidine is a safe and effective analgesic substitute to fentanyl intraoperatively and reduces analgesic requirements postoperatively $[8,9]$. An intraoperative use of dexmedetomidine as a replacement for fentanyl has been shown to reduce opiate use in postoperative period in adults, but analgesic sparing effect in pediatric cases are conflicting and require more research ${ }^{[10,11]}$.

With the above context the present study was designed to assess the efficacy of dexmedetomidine on post-operative sedation and analgesia in paediatric cases undergoing adenotonsillectomy. 


\section{Materials and Methods}

The present prospective study was conducted in the Department of Anesthesiology in association with Department of ENT at MNR Medical College and Hospital, Sangareddy during March 2019 to March 2020. A total 80 pediatric cases undergoing adenotonsillectomy between age group 4-9 years belong to ASA grade I and II were included. Cases with systemic disorders sleep disorders and congenital disorders were excluded. Informed consent was obtained from all study participants and study protocol was approved by institutional ethics committee. Study participants were randomly divided into 2 groups i.e. group 1 with $1 \mathrm{mcg} / \mathrm{kg}$ Dexmeditomidine and group 2 with 2 $\mathrm{mcg} / \mathrm{kg}$ fentanyl. All the participants were administered orally with syrup triclofos $75 \mathrm{mg} / \mathrm{kg}$ before surgery. Cases were pre-oxygenated with $100 \%$ oxygen and induced with IV Propofol $2 \mathrm{mg} / \mathrm{kg}$ followed by Inj succinycholine $1.5 \mathrm{mg} / \mathrm{kg}$. Patient is then intubated nasally with appropriate endotracheal tube and maintained with mask O2+N20 (1:1) + Sevoflurane $2 \%$ through closed circuit with atracurium $0.3 \mathrm{mg} / \mathrm{kg}$ loading dose and $0.1 \mathrm{mg} / \mathrm{kg}$ as supplemental dose every $15 \mathrm{~min}$. The time at which inhalational agent is stopped before end of procedure is noted. Parameters like heart rate, systolic blood pressure (SBP), Diastolic blood pressure (DBP), requirement of postoperative analgesia and sedation and postoperative complications were monitored.
Ramsay sedation score and CHEOPS score (Children's Hospital of Eastern Ontario Pain Scale score) were noted during stay in post-operative recovery room at every 5 minute interval for the first 15 minutes and then for every 15 minutes. The SPSS version 23 software was used to carry out statistical analysis relevant to the study. A p-value of less than 0.05 was considered statistically significant.

\section{Results}

A total 80 pediatric cases undergoing adenotonsillectomy between age group 4-9 years belong to ASA grade I and II were included. The study participants were randomly divided in to 2 groups. Group 1 consists of 40 cases administered with $1 \mathrm{mcg} / \mathrm{kg}$ Dexmeditomidine and group 2 consists of 40 cases administered with $2 \mathrm{mcg} / \mathrm{kg}$ fentanyl. Among the study participants males $(60 \%$ in group $1,55 \%$ in group 2) are more than females (40\% in group 1, $45 \%$ in group 2).

Table 1: Age wise distribution and duration for opening eye in study cases.

\begin{tabular}{|c|c|c|c|}
\hline \multirow{2}{*}{ Parameters } & Group 1 & Group 2 & \multirow{2}{*}{ p-value } \\
\cline { 2 - 4 } & Mean \pm SD & Mean \pm SD & \\
\hline Age & $4.79 \pm 1.01$ & $4.86 \pm 1.32$ & 0.442 \\
\hline Duration for opening eye & $6.23 \pm 0.94$ & $4.45 \pm 1.18$ & 0.002 \\
\hline
\end{tabular}

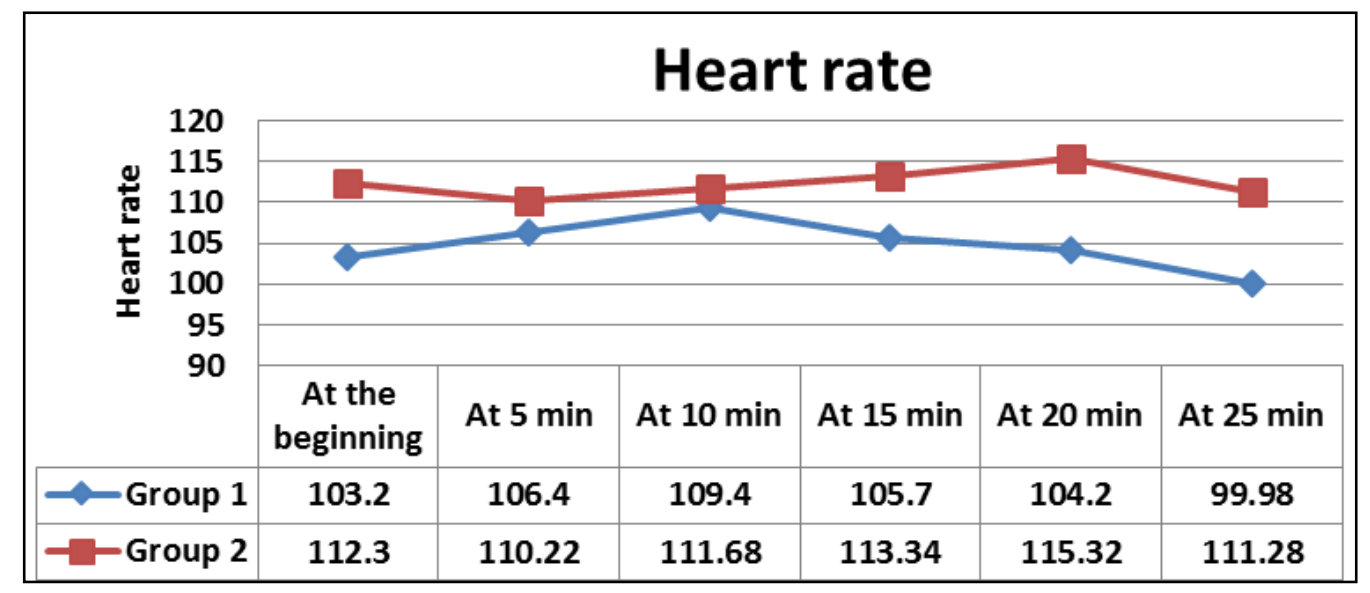

Graph 1: Mean heart rate of study participants.

The mean heart rate was significant between two study groups at the beginning, $5 \mathrm{~min}, 15 \mathrm{~min}, 20 \mathrm{~min}$ and at 25 min $(p<0.005)$. The mean systolic blood pressure was significant between group $1 \& 2$ at $5 \mathrm{~min}, 15 \mathrm{~min}, 20 \mathrm{~min}$ and $25 \min (p<0.005)$. The mean diastolic blood pressure was significant between group $1 \& 2$ during at all-time intervals $(p<0.005)$.

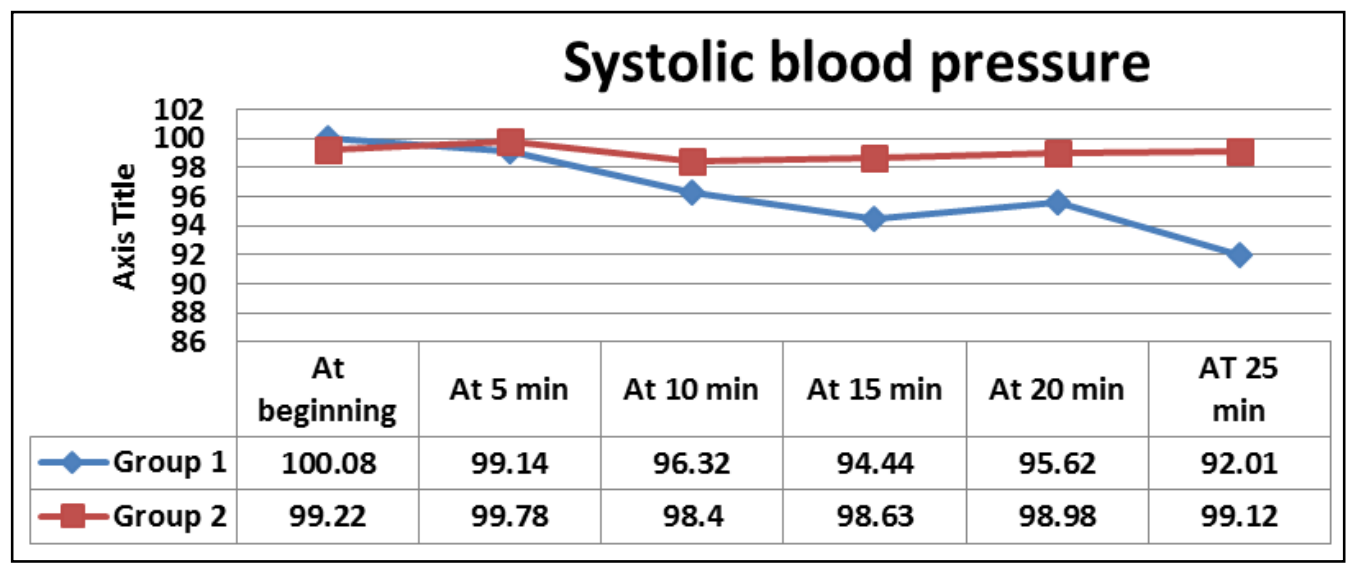

Graph 2: Mean systolic blood pressure (SBP) in study participants. 


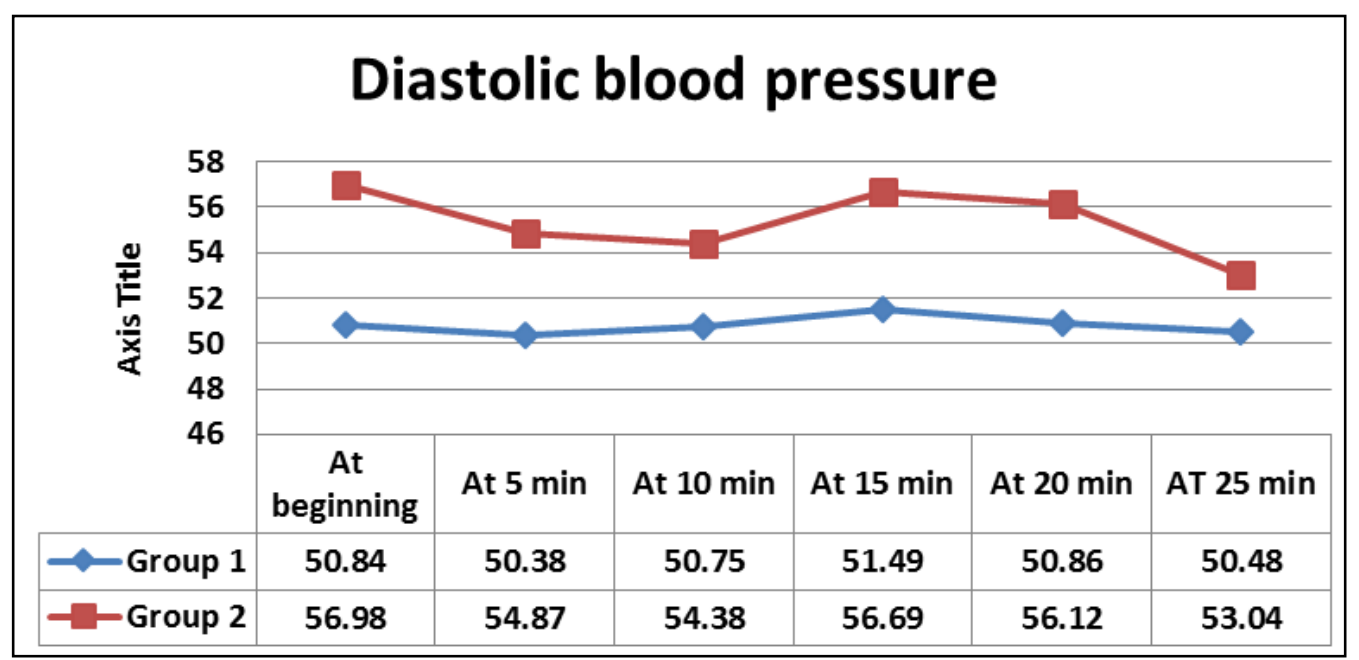

Graph 2: Mean diastolic blood pressure (DBP) in study participants.

Table 2: Ramsay sedation score and CHEOPS score in study participants.

\begin{tabular}{|c|c|c|c|c|c|c|}
\hline & \multicolumn{3}{|c|}{ Ramsay sedation score } & \multicolumn{3}{|c|}{ CHEOPS score } \\
\hline & Group 1 & Group 2 & \multirow{2}{*}{ p value } & Group 1 & Group 2 & \multirow{2}{*}{$p$ value } \\
\hline & Mean \pm SD & Mean \pm SD & & Mean \pm SD & Mean \pm SD & \\
\hline At beginning & $6.10 \pm 0.68$ & $5.14 \pm 0.56$ & 0.002 & $8.74 \pm 0.64$ & $9.08 \pm 0.68$ & 0.003 \\
\hline At $5 \mathrm{~min}$ & $5.73 \pm 0.56$ & $4.58 \pm 0.48$ & 0.002 & $8.22 \pm 0.53$ & $8.65 \pm 0.76$ & 0.002 \\
\hline At $10 \mathrm{~min}$ & $4.38 \pm 0.52$ & $3.74 \pm 0.48$ & 0.001 & $7.66 \pm 0.28$ & $7.97 \pm 0.63$ & 0.014 \\
\hline At $15 \mathrm{~min}$ & $3.98 \pm 0.44$ & $3.04 \pm 0.22$ & 0.003 & $7.49 \pm 0.28$ & $7.63 \pm 0.44$ & 0.228 \\
\hline At $30 \mathrm{~min}$ & $3.32 \pm 0.18$ & $2.66 \pm 0.18$ & 0.565 & $7.04 \pm 0.34$ & $7.54 \pm 0.39$ & 0.298 \\
\hline At $45 \mathrm{~min}$ & $3.04 \pm 0.20$ & $2.31 \pm 0.15$ & 0.002 & $6.95 \pm 0.28$ & $7.38 \pm 0.22$ & 0.005 \\
\hline At $60 \mathrm{~min}$ & $2.75 \pm 0.12$ & $2.08 \pm 0.09$ & 0.412 & $6.66 \pm 0.42$ & $7.19 \pm 0.20$ & 0.003 \\
\hline At $75 \mathrm{~min}$ & $2.45 \pm 0.12$ & $2.01 \pm 0.13$ & 0.328 & $6.61 \pm 0.36$ & $7.08 \pm 0.17$ & 0.452 \\
\hline At $90 \mathrm{~min}$ & $2.20 \pm 0.08$ & $1.89 \pm 0.15$ & 0.284 & $6.48 \pm 0.48$ & $6.86 \pm 0.22$ & 0.360 \\
\hline At $105 \mathrm{~min}$ & $1.84 \pm 0.18$ & $1.72 \pm 0.26$ & 0.223 & $6.32 \pm 0.18$ & $6.72 \pm 0.28$ & 0.562 \\
\hline At $120 \mathrm{~min}$ & $1.52 \pm 0.20$ & $1.66 \pm 0.34$ & 0.126 & $6.18 \pm 0.22$ & $6.48 \pm 0.32$ & 0.289 \\
\hline
\end{tabular}

The mean Ramsay sedation score difference was significant between 2 study groups at the beginning, $5 \mathrm{~min}, 10 \mathrm{~min}, 15$ min and 45 min $(p<0.005)$ (Table 2$)$. The mean CHEOPS score difference in the two study groups was statistically significant (Table 2). The requirement of additional rescue doses were noticed more in group 2 . There were no severe adverse events in group 1, whereas in group 2, nausea and vomiting was observed in $10 \%$ cases.

\section{Discussion}

Adenotonsillectomy is a common surgical procedure in the paediatric age group where postoperative management is crucial due to severe pain affects analgesic consumption, duration of hospital stay and often associated with high incidences of anxiety, obstructive symptoms and hypoxemia $[2,12]$. The present study was designed to assess the efficacy of dexmedetomidine on post-operative sedation and analgesia in paediatric cases undergoing adenotonsillectomy. A total 80 pediatric cases undergoing adenotonsillectomy between age group 4-9 years belong to ASA grade I and II were recruited. The mean age in group 1 was 4.79 years and in group was 4.86 years. The mean duration for opening eyes in group 1 was 6.23 and whereas in group 2 were 4.45 . A study by Hala $\mathrm{S}$ et al. on 84 cases, aged from 5-12 years with mean age 8.26 years. There were significant differences between age, gender and duration of the surgery ${ }^{[14]}$. A study by Jun-Li Cao et al. found that the time to wake and time to extubation were prolonged in group dexmedetomidine group as compared with group administered with $0.9 \%$ saline $(P<0.05)(18)$. A study by
Sanaa M. Elfawal et al. stated that the time to awakening was significantly shorter dexmedetomidine group than fentanyl group [21]. A meta-analysis by Marco Aurelio Soares Amorim et al. stated duration for wakeup was high in dexmedetomidine group ${ }^{[22]}$.

The mean heart rate was significant between two study groups at the beginning, $5 \mathrm{~min}, 15 \mathrm{~min}, 20 \mathrm{~min}$ and at 25 min $(p<0.005)$. The mean heart rate was less in group 1 throughout the surgery than group 2 (Graph 1). A study by Sharma et al. found significant difference between heart rate and mean blood pressure at preincision and postintubation and every 5 min reading till 30 minutes ${ }^{[13]}$. The mean heart rates were significantly lower in DEX-IV group during and after intravenous infusion ${ }^{[14]}$. A study by Jun-Li Cao et al. found mean intraoperative heart rate was significantly lower in dexmedetomidine group $(P<0.05)$, mean DBP and SBP was not statistically different between study groups ${ }^{[18]}$. The mean systolic blood pressure was significant between group $1 \& 2$ at $5 \mathrm{~min}, 15 \mathrm{~min}, 20 \mathrm{~min}$ and $25 \mathrm{~min}(p<0.005)$. The values of mean SBP in group 1 was high at the beginning, later the values were low compared group 2 (Graph 2 ). The mean diastolic blood pressure was significant between group $1 \& 2$ during at all-time intervals $(p<0.005)$. The mean DBP was less in group 1 throughout the surgery than group 2 (Graph 3). A study by Sanaa M. Elfawal et al. found no significant difference in preoperative heart rate, but there was significantly lower heart rate in dexmedetomidine group than fentanyl group ${ }^{[21]}$. A study by Rabie Soliman and Ali Alshehri found that the mean heart rate and mean arterial blood pressure was decreased after 
induction in both study groups. Dexmedetomidine decresed the incidence of postoperative nausea and vomiting, emergence agitation score ${ }^{[23]}$.

The mean Ramsay sedation score difference was significant between 2 study groups at the beginning, $5 \mathrm{~min}, 10 \mathrm{~min}, 15$ min and 45 min $(p<0.005)$ (Table 2). The mean Ramsay sedation score in the first 240 minutes postoperative was decreased in all the study groups. However, the mean sedation scores were significantly higher in DEX.IV group [14]. The mean CHEOPS score difference in the two study groups was statistically significant (Table 2). The mean CHEOPS score was significantly increased from the beginning to $90^{\text {th }}$ minute ${ }^{[14]}$.

The requirement of additional rescue doses were noticed more in group 2. A study by Sharma et al. found no episodes of excessive sedation, desaturation and any drug related adverse events in dexmedetomidine group ${ }^{[13]}$. There were no severe adverse events in group 1, whereas in group 2 , nausea and vomiting was observed in $10 \%$ cases. Postoperative pain was low in DEX group ${ }^{[14]}$. A study by Belleville JP et al. noticed few respiratory side effects and episodes of obstructive apnea in cases who received high doses of dexmedetomidine ${ }^{[15]}$. A randomized, placebocontrolled Study by Guler G et al. found better pain and agitation scores $(p<0.005)$. However, mean heart rate, mean blood pressure, emergence time and time to extubation were significantly lower and longer respectively ${ }^{[16]}$. A study by Isik B et al. stated that dexmedetomine $1 \mu \mathrm{g} / \mathrm{kg}$ as a single dose was effective in reducing the incidence of agitation The mean agitation scores were also significantly lower in the dexmedetomidine group $(p<0.013)$ at $5,10,15$, and 20 minutes after sevoflurane discontinuation ${ }^{[17]}$. A study by Jun-Li Cao et al. stated that objective pain score was lower in dexmedetomidine group at 15, 30, and 45minutes ${ }^{[18]}$. A study by Yuquan Rao et al. stated that compared with placebo, midazolam, and opioids, dexmedetomidine significantly decreased the incidence of post-anesthesia emergence agitation or delirium in pediatric patients ${ }^{[19]}$. A meta-analysis by Juan Ni et al. stated that dexmedetomidine decreased the incidence of severe pain $(p<0.001)$ and need of rescue drug $(p<0.001)$. However, it increased the time to eye open by 0.98 min compared to placebo (20). A metaanalysis by Marco Aurelio Soares Amorim et al. stated that the use of dexmedetomidine was useful in controlling postoperative agitation, nausea and vomiting ${ }^{[22]}$.

\section{Conclusion}

The results of this study conclude that the addition of dexmedetomidine was effective in maintaining intraoperative heart rate, systolic blood pressure and diastolic blood pressure. Dexmedetomidine is an effective and safe analgesic substitute to fentanyl intra-operatively and reduces the requirement of postoperative rescue opioid. Dexmedetomidine was more effective in preventing emergence agitation, avoiding severe pain and reducing incidence of postoperative nausea and vomiting.

\section{References}

1. Guler G, Akin A, Tosun Z, Ors S, Esmaoglu A, Boyaci A, et al. Single-dose dexmedetomidine reduces agitation and provides smooth extubation after pediatric adenotonsillectomy. Paediatr Anaesth 2005;15:762-6.

2. Olutoyin A Olutoye, Chris D Glover, John W Diefenderfer, Michael McGilberry, Matthew M Wyatt,
Deidre R Larrier, et al. The Effect of Intraoperative Dexmedetomidine on Postoperative Analgesia and Sedation in Pediatric Patients Undergoing Tonsillectomy and Adenoidectomy. Pediatric Anesthesiology 2010;111(2):490-495.

3. Sottas CE, Anderson BJ. Dexmedetomidine: The new all-in-one drug in paediatric anaesthesia? Curr Opin Anaesthesiol 2017;30:441-51.

4. Hall JE, Uhrich TD, Barney JA, Arain SR, Ebert TJ Sedative, Amnestic, and analgesic properties of small-dose dexmedetomidine infusions. Anesth Analg 2000;90:699-705.

5. Bhana NL, Goa KL, McClellan KJ. Dexmedetomidine. Drugs 2000;59:263-8.

6. Aiji Boku, Hiroshi Hanamoto, Aiko Oyamaguchi, Mika Inoue, Yoshinari Morimoto, Hitoshi Niwa, et al. Effectiveness of dexmedetomidine for emergence agitation in infants undergoing palatoplasty: a randomized controlled trial. Rev Bras Anestesiol 2016;66(1):37-43.

7. Ge DJ, Qi B, Tang G, et al. Intraoperative dexmedetomidine promotes postoperative analgesia in patients after abdominal colectomy: a consortprospective, randomized, controlled clinical trial. Medicine (Baltimore) 2015;94:e1514.

8. Shukry M, Clyde MC, Kalarickal PL, et al. Does dexmedetomidineprevent emergence delirium in children after sevoflurane-basedgeneral anesthesia? Paediatr Anaesth 2005;15:1098-104.

9. Guler G, Akin A, Tosun Z, et al. Single-dose dexmedeto-midine reduces agitation and provides smooth extubationafter pediatric adenotonsillectomy. Paediatr Anaesth 2005;15:762-6.

10. Patel A, Davidson M, Tran MC, Quraishi H, Schoenberg C, Sant M, et al. Dexmedetomidine infusion for analgesia and prevention of emergence agitation in children with obstructive sleep apnea syndrome undergoing tonsillectomy and adenoidectomy. Anesth Analg 2010;111:1004-1010.

11. Tufanogullari B, White PF, Peixoto MP, Kianpour D, Lacour T, Griffin J, et al. Dexmedetomidine infusion during laparoscopic bariatric surgery: the effect on recovery outcome variables. Anesth Analg 2008;106:1741-1748.

12. Cortney MJ, Cabraal D. Tramadol vs diclofenac for posttonsillectomy analgesia. Arch Otolaryngol Head Neck Surg 2001;127(4):385-8.

13. Sharma K, Kumar M, Gandhi R. Effect of single-dose dexmedetomidine on intraoperative hemodynamics and postoperative recovery during pediatric adenotonsillectomy. Anesth Essays Res 2019;13:63-7.

14. Hala S Abdel-Ghaffar, Alaa Kamel Abdel-Haleem. Efficacy and safety of intraoperative dexmedetomidine in pediatric posttonsillectomy pain: Peritonsillar versus intravenous administration, Egyptian Journal of Anaesthesia 2011;27(4):219-225.

15. Belleville JP, Ward DS, Bloor BC, Maze M. Effects of intravenous dexmedetomidine in humans. I. Sedation, ventilation, and metabolic rate. Anesthesiology 1992;77:1125-33.

16. Guler G, Akin A, Tosun Z, et al. Single-dose dexmedetomidine reduces agitation and provides smooth extubation after pediatric adenotonstillectomy. Pediatr Anesth 2005; 15: 762-6. 
17. Isik B, Arslan M, Tunga AD, et al. Dexmedetomidine decreases emergence agitation in pediatric patients after sevoflurane anesthesia without surgery. Pediatr Anesth 2006; 16: 748-53.

18. Jun-Li Cao, Yu-Ping Pei, Jing-Qiu Wei, Yue-Ying Zhang. Effects of intraoperative dexmedetomidine with intravenous anesthesia on postoperative emergence agitation/delirium in pediatric patients undergoing tonsillectomy with or without Adenoidectomy. Medicine. 2016; 95(49): 1-6.

19. Yuquan Rao, Zeng R, Jiang X, Li J and Wang X. The Effect of Dexmedetomidine on Emergence Agitation or Delirium in Children after Anesthesia-A Systematic Review and Meta-Analysis of Clinical Studies. Front. Pediatr.202; 8:329.

20. Juan Ni, Wei J, Yao Y, Jiang X, Luo L, Luo D. Effect of Dexmedetomidine on Preventing Postoperative Agitation in Children: A Meta-Analysis. PLoS ONE. 2015; 10(5):1-13.

21. Sanaa M. Elfawal, Abeer M. Eldeek, Manal M. Kamal. Dexmedetomidine infusion versus fentanyl for analgesia and prevention of emergence agitation and delirium in children undergoing adenotonsillectomy. Ain-Shams Journal of Anaesthesiology 2016;9:485492.

22. Marco Aurelio Soares Amorim, Catia Sousa Goveia, Edno Magalhaes, Luís Claudio Araujo Ladeira, Larissa Goveia Moreira, Denismar Borges de Miranda. Effect of dexmedetomidine in children undergoing general anesthesia with sevoflurane: a meta-analysis. Rev Bras Anestesiol 2017;67(2):193-198.

23. Rabie Soliman, Ali Alshehri. Effect of dexmedetomidine on emergence agitation in children undergoing adenotonsillectomy under sevoflurane anesthesia: A randomized controlled study. Egyptian Journal of Anaesthesia 2015;31:283-289.

24. Mother's possess some knowledge regarding prevention and home care management of chickenpox in children. 